\title{
STANLEY DEPTH OF POWERS OF THE EDGE IDEAL OF A FOREST
}

\author{
M. R. POURNAKI, S. A. SEYED FAKHARI, AND S. YASSEMI
}

(Communicated by Irena Peeva)

\begin{abstract}
Let $\mathbb{K}$ be a field and $S=\mathbb{K}\left[x_{1}, \ldots, x_{n}\right]$ be the polynomial ring in $n$ variables over the field $\mathbb{K}$. Let $G$ be a forest with $p$ connected components $G_{1}, \ldots, G_{p}$ and let $I=I(G)$ be its edge ideal in $S$. Suppose that $d_{i}$ is the diameter of $G_{i}, 1 \leq i \leq p$, and consider $d=\max \left\{d_{i} \mid 1 \leq i \leq p\right\}$. Morey has shown that for every $t \geq 1$, the quantity $\max \left\{\left\lceil\frac{d-t+2}{3}\right\rceil+p-1, p\right\}$ is a lower bound for $\operatorname{depth}\left(S / I^{t}\right)$. In this paper, we show that for every $t \geq 1$, the mentioned quantity is also a lower bound for $\operatorname{sdepth}\left(S / I^{t}\right)$. By combining this inequality with Burch's inequality, we show that any sufficiently large powers of edge ideals of forests are Stanley. Finally, we state and prove a generalization of our main theorem.
\end{abstract}

\section{INTRODUCTION}

Let $\mathbb{K}$ be a field and $S=\mathbb{K}\left[x_{1}, \ldots, x_{n}\right]$ be the polynomial ring in $n$ variables over the field $\mathbb{K}$. Let $I$ be a monomial ideal in $S$. We denote the $\mathbb{K}$-linear subspace of $S$ generated by all monomials which do not belong to $I$ by $I^{c}$. Therefore $S=I \oplus I^{c}$, and $S / I \cong I^{c}$ as $\mathbb{K}$-linear spaces. Let $u \in S$ be a monomial and $Z \subseteq\left\{x_{1}, \ldots, x_{n}\right\}$. The $\mathbb{K}$-subspace $u \mathbb{K}[Z]$ whose basis consists of all monomials $u v$ with $v \in \mathbb{K}[Z]$ is called a Stanley space of dimension $|Z|$. Here, as usual, $|Z|$ denotes the number of elements of $Z$. A decomposition $\mathcal{D}$ of $I^{c}$ as a finite direct sum of Stanley spaces is called a Stanley decomposition of $S / I$. The minimum dimension of a Stanley space in $\mathcal{D}$ is called the Stanley depth of $\mathcal{D}$ and is denoted by $\operatorname{sdepth}(\mathcal{D})$. The quantity

$$
\operatorname{sdepth}(S / I):=\max \{\operatorname{sdepth}(\mathcal{D}) \mid \mathcal{D} \text { is a Stanley decomposition of } S / I\}
$$

is called the Stanley depth (sdepth for short) of $S / I$. For a reader-friendly introduction to sdepth, we refer the reader to [5]. Stanley [9] made a conjecture on Stanley decompositions of $\mathbb{Z}^{n}$-graded modules. In the special case when the $\mathbb{Z}^{n}$ graded module is $S / I$, where $I$ is a monomial ideal of $S$, the conjecture says that $\operatorname{sdepth}(S / I) \geq \operatorname{depth}(S / I)$. A monomial ideal $I$ of $S$ is called Stanley if it satisfies Stanley's conjecture.

Let $G$ be a forest with $p$ connected components $G_{1}, \ldots, G_{p}$ and let $I=I(G)$ be its edge ideal in $S$. Suppose that $d_{i}$ is the diameter of $G_{i}, 1 \leq i \leq p$, and consider

Received by the editors August 25, 2011 and, in revised form, December 10, 2011.

2010 Mathematics Subject Classification. Primary 13C15, 05E99; Secondary 13C13.

Key words and phrases. Edge ideal, monomial ideal, Stanley depth, Stanley conjecture.

The research of the first and third authors was partially supported by grants from IPM (No. 90130073 and No. 90130214). 
$d=\max \left\{d_{i} \mid 1 \leq i \leq p\right\}$. Morey has shown that for every $t \geq 1$, the inequality

$$
\operatorname{depth}\left(S / I^{t}\right) \geq \max \left\{\left\lceil\frac{d-t+2}{3}\right\rceil+p-1, p\right\}
$$

holds true. Assuming Stanley's conjecture and combining it with Morey's inequality, one should expect that for every $t \geq 1$,

$$
\operatorname{sdepth}\left(S / I^{t}\right) \geq \max \left\{\left\lceil\frac{d-t+2}{3}\right\rceil+p-1, p\right\} .
$$

In this paper, we prove the latter inequality. By combining this inequality with Burch's inequality, we show that any sufficiently large powers of edge ideals of forests are Stanley. Finally, we state and prove a generalization of our main theorem.

We remark that the proofs of the main theorem and its extension are minor modifications of the original proofs for depth, replacing depth by sdepth and the depth lemma by [7, Lemma 2.2]. Nevertheless, here we give complete proofs in order to make the treatment clear and self-contained.

\section{The MAIN RESUlts}

Let $G$ be a graph with vertex-set $V(G)=\left\{v_{1}, \ldots, v_{n}\right\}$. For a vertex $v_{i}$, the neighbor set of $v_{i}$ is $N\left(v_{i}\right)=\left\{v_{j} \mid v_{i} v_{j}\right.$ is an edge of $\left.G\right\}$. The vertex $v_{i}$ is called a leaf if $N\left(v_{i}\right)$ is a singleton and is called isolated if $N\left(v_{i}\right)$ is empty. There are two types of operations performed on a graph that produce smaller, related graphs that are referred to as minors of $G$. The one used here is the deletion, denoted by $G \backslash v$, which is formed by removing the vertex $v$ from the vertex-set of $G$ and deleting any edge in $G$ that contains $v$. Paths are special types of graphs. For $n \geq 2$, a path $P_{n}$ of length $n-1$ is a set of $n$ distinct vertices $x_{1}, \ldots, x_{n}$ together with $n-1$ edges $x_{i} x_{i+1}$ for $1 \leq i \leq n-1$. The diameter of a connected graph is the maximum distance between any two vertices. Here, the distance between two vertices is the minimum length of a path connecting the vertices. Thus if the diameter of a graph $G$ is $d$, then there exist vertices $u$ and $v$ of $G$ and a path $P_{d+1}$ of length $d$ connecting $u$ and $v$ such that no path of length less than $d$ exists between $u$ and $v$. Such a path will be referred to as a path realizing the diameter of $G$.

Proposition 2.1. Let $P_{n}$ be a path and $I\left(P_{n}\right)$ be its edge ideal. Then we have

$$
\operatorname{sdepth}\left(S / I\left(P_{n}\right)\right) \geq\left\lceil\frac{n}{3}\right\rceil .
$$

Proof. For $n$ with $n \leq 3$, the assertion holds by [3, Theorem 1.4]. We therefore assume that $n \geq 4$. Consider the following short exact sequence:

$$
0 \longrightarrow S /\left(I\left(P_{n}\right): x_{n-1}\right) \longrightarrow S / I\left(P_{n}\right) \longrightarrow S /\left(I\left(P_{n}\right), x_{n-1}\right) \longrightarrow 0 .
$$

Since $\left(I\left(P_{n}\right): x_{n-1}\right)=\left(I\left(P_{n-3}\right), x_{n-2}, x_{n}\right)$, by induction and [6, Theorem 1.1] we obtain that

$$
\begin{aligned}
\operatorname{sdepth}\left(S /\left(I\left(P_{n}\right): x_{n-1}\right)\right) & =\operatorname{sdepth}\left(S^{\prime}\left[x_{n-1}\right] / I\left(P_{n-3}\right)\right) \\
& =\operatorname{sdepth}\left(S^{\prime} / I\left(P_{n-3}\right)\right)+1 \\
& \geq\left\lceil\frac{n-3}{3}\right\rceil+1=\left\lceil\frac{n}{3}\right\rceil
\end{aligned}
$$


where $S^{\prime}=\mathbb{K}\left[x_{1}, x_{2}, \ldots, x_{n-3}\right]$. Since $\left(I\left(P_{n}\right), x_{n-1}\right)=\left(I\left(P_{n-2}\right), x_{n-1}\right)$, similarly we obtain that

$$
\operatorname{sdepth}\left(S /\left(I\left(P_{n}\right), x_{n-1}\right)\right)=\operatorname{sdepth}\left(S^{\prime}\left[x_{n-2}\right] / I\left(P_{n_{2}}\right)\right)+1 \geq\left\lceil\frac{n-3}{3}\right\rceil+1 .
$$

Now [7, Lemma 2.2] implies that $\operatorname{sdepth}\left(S / I\left(P_{n}\right)\right) \geq\left\lceil\frac{n}{3}\right\rceil$, as required.

By applying Proposition 2.1 and [4, Lemma 2.8] we conclude the following corollary.

Corollary 2.2. The edge ideal of every path is a Stanley ideal.

The sdepth formula for a path given by Proposition 2.1 can be extended to a formula that gives a lower bound for the sdepth of a tree. Note that since the diameter is the maximum distance between vertices, a path realizing the diameter of a tree must connect two leaves of the tree, where a leaf is a vertex with a unique neighbor.

Proposition 2.3. Let $G$ be a tree of diameter $d$ and $I=I(G)$ its edge ideal. Then we have

$$
\operatorname{sdepth}(S / I) \geq\left\lceil\frac{d+1}{3}\right\rceil
$$

Proof. If $d \leq 2$, then $\left\lceil\frac{d+1}{3}\right\rceil=1$, and so the result follows from [3. Theorem 1.4]. We therefore suppose that $d \geq 3$. In this case, the proof is essentially the proof of [4, Proposition 2.9], with depth replaced by sdepth and the depth lemma replaced by [7, Lemma 2.2]. We provide the proof for completeness.

Let $u$ and $v$ be the vertices of $G$ such that the distance between $u$ and $v$ is $d$, and let $P_{d+1}$ be a path connecting $u$ and $v$ that realizes the diameter of $G$. Then $u$ is a leaf, and so we may consider $y$ as the unique neighbor of $u$. Therefore we obtain that $(I, y)=(J, y)$, where $J$ is the edge ideal of the minor $G^{\prime}$ of $G$ formed by deleting $y$. Note that the diameter of $G^{\prime}$ is at least $d-2$ and also that $u$ is isolated in $G^{\prime}$. Thus if $S^{\prime}$ is the polynomial ring formed by deleting $u$, then $\operatorname{sdepth}(S /(I, y))=\operatorname{sdepth}\left(S^{\prime}[u] /(J, y)\right)$. Now [6. Theorem 1.1] implies that $\operatorname{sdepth}(S /(I, y))=\operatorname{sdepth}\left(S^{\prime} /(J, y)\right)+1$, and again by [6, Theorem 1.1] and using induction we conclude that

$$
\operatorname{sdepth}(S /(I, y)) \geq\left\lceil\frac{d-2+1}{3}\right\rceil+1 \geq\left\lceil\frac{d+1}{3}\right\rceil .
$$

Now consider $(I: y)=(L, N(y))$, where $L$ is the ideal of the minor $G^{\prime \prime}$ of $G$ formed by deleting the variables in $N(y)$. Let $S^{\prime \prime}$ be the polynomial ring formed by deleting the variables in $y \cup N(y)$. Then the diameter of $G^{\prime \prime}$ is at least $d-3$ and $y$ is an isolated vertex, and so

$$
\operatorname{sdepth}(S /(I: y))=\operatorname{sdepth}\left(S^{\prime \prime}[y] / L\right) \geq\left\lceil\frac{d-3+1}{3}\right\rceil+1=\left\lceil\frac{d+1}{3}\right\rceil
$$

by induction and [6, Theorem 1.1].

We now conclude the proposition by applying [7, Lemma 2.2] to the following short exact sequence:

$$
0 \longrightarrow S /(I: y) \longrightarrow S / I \longrightarrow S /(I, y) \longrightarrow 0 .
$$


The goal of this paper is to use graph invariants to provide lower bounds on the sdepth of the powers of the edge ideal of a forest. When the graph is a tree or forest, a lower bound for the sdepth of any power will be given in Theorem 2.7. Since the proof makes repeated use of applying [7, Lemma 2.2] to a pair of sequences, we first state and prove a lemma for simplification.

Lemma 2.4. Let $G$ be a graph and $I=I(G)$ be its edge ideal. If $z_{1}$ and $z_{2}$ are vertices of $G$ and for some $s \geq 0, \operatorname{sdepth}\left(S /\left(I^{t}: z_{1} z_{2}\right)\right) \geq s, \operatorname{sdepth}\left(S /\left(I^{t}, z_{1}\right)\right) \geq s$ and $\operatorname{sdepth}\left(S /\left(\left(I^{t}: z_{1}\right), z_{2}\right)\right) \geq s$, then $\operatorname{sdepth}\left(S / I^{t}\right) \geq s$.

Proof. The proof is essentially the proof of [4, Lemma 3.1] with depth replaced by sdepth and the depth lemma replaced by [7, Lemma 2.2]. We provide the proof for completeness. Applying [7, Lemma 2.2] to the short exact sequence

$$
0 \longrightarrow S /\left(I^{t}: z_{1} z_{2}\right) \longrightarrow S /\left(I^{t}: z_{1}\right) \longrightarrow S /\left(\left(I^{t}: z_{1}\right), z_{2}\right) \longrightarrow 0
$$

yields that $\operatorname{sdepth}\left(S /\left(I^{t}: z_{1}\right)\right) \geq s$. Now again applying [7, Lemma 2.2] to the short exact sequence

$$
0 \longrightarrow S /\left(I^{t}: z_{1}\right) \longrightarrow S / I^{t} \longrightarrow S /\left(I^{t}, z_{1}\right) \longrightarrow 0
$$

implies that $\operatorname{sdepth}\left(S / I^{t}\right) \geq s$.

As a first step toward determining the sdepth of powers of edge ideals of trees and forests, we may now determine a lower bound on the sdepth of the powers of the ideal of a path.

Proposition 2.5. Let $P_{n}$ be a path and $I\left(P_{n}\right)$ be its edge ideal. If $n \geq 2$ and $t \geq 1$, then we have

$$
\operatorname{sdepth}\left(S / I\left(P_{n}\right)^{t}\right) \geq \max \left\{\left\lceil\frac{n-t+1}{3}\right\rceil, 1\right\} .
$$

Proof. Note that by [3, Theorem 1.4] and [8, Theorem 5.9], for $n \geq 2$ and $t \geq 1$ we have $\operatorname{sdepth}\left(S / I\left(P_{n}\right)^{t}\right) \geq 1$. We therefore prove that

$$
\operatorname{sdepth}\left(S / I\left(P_{n}\right)^{t}\right) \geq\left\lceil\frac{n-t+1}{3}\right\rceil .
$$

We use induction on $n$ and $t$ to prove this latter inequality. Note that by 3 , Theorem 1.4] and [8, Theorem 5.9] the result holds for $n \leq 3$ and for all $t$. Also, by Proposition 2.1, the result holds for $t=1$ and for all $n$. We therefore assume that $n \geq 4$ and $t \geq 2$. By this assumption, the proof is the same as the proof of [4, Proposition 3.2], with depth replaced by sdepth, the depth lemma replaced by [7, Lemma 2.2] and [4, Lemma 3.1] replaced by Lemma 2.4

The following lemma is an immediate consequence of [7, Theorem 3.1].

Lemma 2.6. Let $G=G_{1} \cup G_{2}$ be the disjoint union of two graphs $G_{1}$ and $G_{2}$. Then we have

$$
\operatorname{sdepth}(S / I(G)) \geq \operatorname{sdepth}\left(S^{\prime} / I\left(G_{1}\right)\right)+\operatorname{sdepth}\left(S^{\prime \prime} / I\left(G_{2}\right)\right),
$$

where $S^{\prime}$ is the polynomial ring over $\left|V\left(G_{1}\right)\right|$ variables, $S^{\prime \prime}$ is the polynomial ring over $\left|V\left(G_{2}\right)\right|$ variables and $S$ is the polynomial ring over $|V(G)|$ variables.

We are now ready to prove the main theorem of this paper. Note that isolated vertices will not be considered as connected components of $G$. 
Theorem 2.7. Let $G$ be a forest with $p$ connected components $G_{1}, \ldots, G_{p}$ and let $I=I(G)$ be its edge ideal in $S$. Suppose that $d_{i}$ is the diameter of $G_{i}, 1 \leq i \leq p$, and consider $d=\max \left\{d_{i} \mid 1 \leq i \leq p\right\}$. Then for every $t \geq 1$ we have

$$
\operatorname{sdepth}\left(S / I^{t}\right) \geq \max \left\{\left\lceil\frac{d-t+2}{3}\right\rceil+p-1, p\right\} .
$$

Proof. To prove the theorem we use induction on $t$ and $n$, where $n$ is the number of nonisolated vertices of $G$. Without loss of generality, we may assume $d=d_{1}$. For $t=1$ and $p=1$ the result follows from Proposition 2.3. For $t=1$ and any $p \geq 2$ the result follows from Lemma 2.6] and Proposition 2.3. Thus the result holds for $t=1$ and every $n$. Now assume that $t \geq 2$. If $n=2$, then $G=P_{2}, d=1$, and the result holds for all $t$ by Proposition 2.5. Assume $n \geq 3$. In this case, the proof is essentially the proof of [4, Theorem 3.4], with depth replaced by sdepth, the depth lemma replaced by [7, Lemma 2.2] and [4, Lemma 3.1] replaced by Lemma 2.4. We provide the proof for completeness.

Fix a path $P_{d+1}$ in $G$ realizing the diameter, let $x_{1}$ be an endpoint of this path (and thus a leaf of $G$ ), let $y$ be its unique neighbor, and let $N(y)=\left\{x_{1}, \ldots, x_{r}\right\}$ be the neighbors of $y$. Note that $r \geq 1$ and $r$ is finite. Note also that by [4, Lemma 3.3], at most one of the $x_{i}$ 's is not a leaf. Without loss of generality, assume that $x_{i}$ is a leaf for $1 \leq i<r$. Let $I_{j}$ be the ideal of the minor of $G$ formed by deleting $x_{1}, \ldots, x_{j}$ for every $1 \leq j \leq r$. Let $S_{j}=\mathbb{K}\left[x_{j+1}, \ldots, x_{n-1}, y\right]$ be the subring of $S$ excluding $x_{1}, \ldots, x_{j}$ and let $S_{j}^{\prime}=\mathbb{K}\left[x_{j+1}, \ldots, x_{n-1}\right]$. Note that for every $j, I_{j} \subseteq S_{j}$ is the edge ideal of a graph involving fewer than $n$ vertices. To use Lemma 2.4 to find the sdepth of $S / I^{t}$, the sdepths of three ideals must be checked. For simplification, let

$$
s=\max \left\{\left\lceil\frac{d-t+2}{3}\right\rceil+p-1, p\right\} .
$$

Since $x_{1}$ is a leaf, by [4, Lemma 2.10], $\left(I^{t}: x_{1} y\right)=I^{t-1}$, and so by induction on $t$ we have

$$
\operatorname{sdepth}\left(S /\left(I^{t}: x_{1} y\right)\right) \geq \max \left\{\left\lceil\frac{d-(t-1)+2}{3}\right\rceil+p-1, p\right\} \geq s .
$$

To find the sdepth of the second ideal, note that $\left(I^{t}, y\right)=\left(J^{t}, y\right)$, where $J$ is the edge ideal of the minor $G^{\prime}$ of $G$ formed by deleting $y$. Then $G^{\prime}$ is again a forest with fewer than $n$ vertices, it has at least $p-1$ connected components, and the generators of $J$ lie in $S_{1}^{\prime}$. Thus by induction, $\operatorname{sdepth}\left(S_{1}^{\prime} / J^{t}\right) \geq p-1$, and so we have

$$
\operatorname{sdepth}\left(S /\left(I^{t}, y\right)\right)=\operatorname{sdepth}\left(S_{1}\left[x_{1}\right] /\left(J^{t}, y\right)\right) \geq p-1+1=p .
$$

Suppose $d \leq 3$. Then $\left\lceil\frac{d-t+2}{3}\right\rceil \leq 1$ for every $t \geq 2$, and thus we have $s=p$ and $\operatorname{sdepth}\left(S /\left(I^{t}, y\right)\right) \geq s$.

For $d>3$, note that the number of connected components of $J$ is at least $p$, since $G_{2}, \ldots, G_{p}$ and $d-2 \geq 1$ edges of $P_{d+1}$ survive in $G^{\prime}$. This also implies that the maximal diameter $d^{\prime}$ of a connected component of $G^{\prime}$ is at least $d-2$. Thus by induction on $n$, we conclude that

$$
\operatorname{sdepth}\left(S_{1}\left[x_{1}\right] /\left(J^{t}, y\right)\right) \geq \max \left\{\left\lceil\frac{d-2-t+2}{3}\right\rceil+p-1, p\right\}+1 \geq s .
$$

Thus for every $d$ we have $\operatorname{sdepth}\left(S /\left(I^{t}, y\right)\right) \geq s$. 
Now consider $\left(\left(I^{t}: y\right), x_{1}\right)$. By 4, Lemma 2.5] we have $\left(\left(I^{t}: y\right), x_{1}\right)=\left(\left(I_{1}^{t}: y\right), x_{1}\right)$, where $I_{1}$ is as defined above. Thus

$$
\operatorname{sdepth}\left(S /\left(\left(I^{t}: y\right), x_{1}\right)\right)=\operatorname{sdepth}\left(S_{1} /\left(I_{1}^{t}: y\right)\right) .
$$

Note that if $r=1$, then $d=1$ and $s=p$ as above. Also, for $r=1,\left(I_{1}^{t}: y\right)=I_{1}^{t}$ and $I_{1} \subseteq S_{1}^{\prime}$ is the edge ideal of a forest with fewer than $n$ vertices and $p-1$ connected components. Thus

$$
\operatorname{sdepth}\left(S /\left(\left(I^{t}: y\right), x_{1}\right)\right)=\operatorname{sdepth}\left(S_{1} / I_{1}^{t}\right)=\operatorname{sdepth}\left(S_{1}^{\prime}[y] / I_{1}^{t}\right) \geq p-1+1=p,
$$

and so for $r=1$ we have $\operatorname{sdepth}\left(S /\left(\left(I^{t}: y\right), x_{1}\right)\right) \geq s$.

Now suppose that $r \geq 2$. In this case $d \geq 2$, since $x_{1}, y, x_{r}$ are all vertices included in $P_{d+1}$. To find $\operatorname{sdepth}\left(S_{1} /\left(I_{1}^{t}: y\right)\right)$, we use reverse induction on $r$. First, consider $\left(I_{r}^{t}: y\right)=I_{r}^{t}$. Note that the generators of $I_{r}^{t}$ all lie in $S_{r}^{\prime}$, and so $\operatorname{sdepth}\left(S_{r} / I_{r}^{t}\right)=$ $\operatorname{sdepth}\left(S_{r}^{\prime}[y] / I_{r}^{t}\right)=\operatorname{sdepth}\left(S_{r}^{\prime} / I_{r}^{t}\right)+1$. If $d \leq 3$, then $I_{r} \subseteq S_{r}^{\prime}$ corresponds to a graph with at least $p-1$ connected components, and so by induction on $n$ we have $\operatorname{sdepth}\left(S_{r}^{\prime} / I_{r}^{t}\right) \geq p-1$ and therefore $\operatorname{depth}\left(S_{r} / I_{r}^{t}\right) \geq p=s$. If $d \geq 4$, then $I_{r}$ corresponds to a graph with diameter at least $d-3$ with at least $p$ connected components, and so by induction on $n$ we have

$$
\operatorname{sdepth}\left(S_{r}^{\prime} / I_{r}^{t}\right) \geq \max \left\{\left\lceil\frac{d-3-t+2}{3}\right\rceil+p-1, p\right\}
$$

and thus

$$
\operatorname{sdepth}\left(S_{r} /\left(I_{r}^{t}: y\right)\right)=\operatorname{sdepth}\left(S_{r} / I_{r}^{t}\right)=\operatorname{sdepth}\left(S_{r}^{\prime} / I_{r}^{t}\right)+1 \geq s .
$$

Now assume that $\operatorname{sdepth}\left(S_{j} /\left(I_{j}^{t}: y\right)\right) \geq s$ for some $2 \leq j \leq r$. Consider the short exact sequence

$$
0 \longrightarrow S_{j-1} /\left(I_{j-1}^{t}: y x_{j}\right) \longrightarrow S_{j-1} /\left(I_{j-1}^{t}: y\right) \longrightarrow S_{j-1} /\left(\left(I_{j-1}^{t}: y\right), x_{j}\right) \longrightarrow 0 .
$$

By [4, Lemma 2.5] we have $\left(\left(I_{j}^{t}: y\right), x_{j}\right)=\left(\left(I_{j-1}^{t}: y\right), x_{j}\right)$ and therefore by assumption we obtain that $\operatorname{sdepth}\left(S_{j-1} /\left(\left(I_{j-1}^{t}: y\right), x_{j}\right)\right)=\operatorname{sdepth}\left(S_{j} /\left(I_{j}^{t}: y\right)\right) \geq s$. By 4 , Lemma 2.10], $\left(I_{j-1}^{t}: y x_{j}\right)=I_{j-1}^{t-1}$, since $x_{j}$ is a leaf for $j<r$ and $y$ is a leaf when $j=r$. The diameter of the graph associated to $I_{j-1}$ is at least $d-1$, and so

$$
\begin{aligned}
\operatorname{sdepth}\left(S_{j-1} /\left(I_{j-1}^{t}: y x_{j}\right)\right) & =\operatorname{sdepth}\left(S_{j-1} / I_{j-1}^{t-1}\right) \\
& \geq \max \left\{\left\lceil\frac{d-1-(t-1)+2}{3}\right\rceil+p-1, p\right\}=s
\end{aligned}
$$

by induction. Thus by [7, Lemma 2.2] we have $\operatorname{sdepth}\left(S_{j-1} /\left(I_{j-1}^{t}: y\right)\right) \geq s$. Hence by reverse induction, $\operatorname{sdepth}\left(S_{1} /\left(I_{1}^{t}: y\right)\right) \geq s$.

$\operatorname{Since} \operatorname{sdepth}\left(S /\left(\left(I^{t}: y\right), x_{1}\right)\right)=\operatorname{sdepth}\left(S_{1} /\left(I_{1}^{t}: y\right)\right)$, as above we conclude that $\operatorname{sdepth}\left(S /\left(\left(I^{t}: y\right), x_{1}\right)\right) \geq s$. Thus by applying Lemma 2.4 with $z_{1}=y$ and $z_{2}=x_{1}$, we obtain that $\operatorname{sdepth}\left(S / I^{t}\right) \geq s$, as required.

Let $\mathbb{K}$ be a field and $S=\mathbb{K}\left[x_{1}, \ldots, x_{n}\right]$ be the polynomial ring in $n$ variables over the field $\mathbb{K}$, and let $I \subset S$ be a monomial ideal. A classical result by Burch [2] so that

$$
\min _{t} \operatorname{depth}\left(S / I^{t}\right) \leq n-\ell(I)
$$

where $\ell(I)$ is the analytic spread of $I$, that is, the dimension of $\mathcal{R}(I) / \mathfrak{m} \mathcal{R}(I)$, where $\mathcal{R}(I)=\bigoplus_{n=0}^{\infty} I^{n}=S[I t] \subseteq S[t]$ is the Rees ring of $I$ and $\mathfrak{m}=\left(x_{1}, \ldots, x_{n}\right)$ is the maximal ideal of $S$. By a theorem of Brodmann [1], $\operatorname{depth}\left(S / I^{t}\right)$ is constant 
for large $t$. We call this constant value the limit depth of $I$, and denote it by $\lim _{t \rightarrow \infty} \operatorname{depth}\left(S / I^{t}\right)$. Brodmann improved the Burch inequality by showing that

$$
\lim _{t \rightarrow \infty} \operatorname{depth}\left(S / I^{t}\right) \leq n-\ell(I) .
$$

Corollary 2.8. Let $G$ be a forest. Then there exists a positive integer $k_{0}$ such that for every $k \geq k_{0}$, the ideal $I^{k}$ is Stanley.

Proof. One can easily check that for every forest $G$, the analytic spread of $I(G)$ is equal to $n-p$, where $p$ is the number of connected components of $G$ (see also [10, Page 50]). Therefore by the minimum value given in [4, Theorem 3.4] and the argument above, $\operatorname{depth}\left(S / I^{t}\right)=p$, for sufficiently large $k$. Now Theorem 2.7 completes the proof.

\section{Appendix: A generalization of the main theorem}

The proof of Theorem 2.7 depends heavily on the existence of a vertex $y$ such that at most one neighbor is not a leaf. A careful examination of [4, Lemma 3.3] guarantees that any tree with diameter $d \geq 3$ will contain at least two such vertices that are not themselves leaves, namely the neighbors of the two leaves of a path realizing the diameter. Call a vertex $v$ of $G$ a near leaf of $G$ if $v$ is not a leaf and $N(v)$ contains at most one vertex that is not a leaf. Let $q$ denote the number of near leaves of $G$. Then the bound given in Theorem 2.7 can be strengthened using essentially the same proof. However, a strengthening of Proposition 2.3 is needed.

Lemma 3.1. Let $G$ be a tree with diameter $d \geq 1$ and $q$ near leaves, and let $I=I(G)$ be its edge ideal. Then we have

$$
\operatorname{sdepth}(S / I) \geq\left\lceil\frac{d+q-1}{3}\right\rceil .
$$

Proof. For $q \leq 2$ the lemma holds by Proposition 2.3, and so we assume that $q \geq 3$. Note that for a connected graph, if two near leaves are adjacent, then we obtain that $d=3$ and $q=2$, since all other vertices must be leaves. Thus for $q \geq 3$, no neighbor of a near leaf is a near leaf. Let $P_{d+1}$ be a path realizing the diameter of $G$ with vertices $x_{1}, x_{2}, \ldots, x_{d+1}$. In this stage, we continue the proof as the proof of [4, Lemma 3.6], with depth replaced by sdepth and the depth lemma replaced by [7, Lemma 2.2].

Corollary 3.2. Let $G$ be a forest with $p$ connected components $G_{1}, \ldots, G_{p}$ and let $I=I(G)$ be its edge ideal in $S$. Suppose that $d_{i}$ is the diameter of $G_{i}, 1 \leq i \leq p$, and consider $d=\max \left\{d_{i} \mid 1 \leq i \leq p\right\}$. If $q$ is the number of near leaves of a component of diameter $d$, then for every $t \geq 1$ we have

$$
\operatorname{sdepth}\left(S / I^{t}\right) \geq \max \left\{\left\lceil\frac{d-t+q}{3}\right\rceil+p-1, p\right\} .
$$

Proof. The proof is essentially the proof of [4, Corollary 3.7], with depth replaced by sdepth and [4, Lemma 3.1] replaced by Lemma 2.4. We provide the proof for completeness.

As before, assume that $d=d_{1}$ and $q$ is the number of near leaves of $G_{1}$. When counting near leaves in this proof, only those in minors of $G_{1}$ will be considered. For simplification, let

$$
s=\max \left\{\left\lceil\frac{d-t+q}{3}\right\rceil+p-1, p\right\} .
$$


If $d \leq 3$, then $q \leq 2$, and so the result holds by Theorem 2.7. When $t=1$, the result follows from Lemma 3.1] [3, Theorem 1.4] and Lemma 2.6, Fix the notation as in Theorem 2.7 and assume $q \geq 3$ and $d \geq 4$. Then $\left(I^{t}: x_{1} y\right)=I^{t-1}$ as before, and

$$
\operatorname{sdepth}\left(S /\left(I^{t}: x_{1} y\right)\right) \geq \max \left\{\left\lceil\frac{d-t+1+q}{3}\right\rceil+p-1, p\right\} \geq s
$$

by induction on $t$. Now $\left(I^{t}, y\right)=\left(J^{t}, y\right)$, where $J$ is the edge ideal of $G^{\prime}$. Note that the diameter of $G^{\prime}$ is at least $d-2, G^{\prime}$ has at least $q-1$ near leaves, and $x_{1}$ is an isolated vertex. Thus by induction on $n$,

$$
\operatorname{sdepth}\left(S /\left(I^{t}, y\right)\right) \geq \max \left\{\left\lceil\frac{d-2-t+q-1}{3}\right\rceil+p-1, p\right\}+1 \geq s,
$$

as before.

As in the proof of Theorem 2.7 since $d \geq 4$, we have $r \geq 2$ and $\left(I_{r}^{t}: y\right)=I_{r}^{t}$. Note that the diameter of the graph corresponding to $I_{r}$ is at least $d-3$. Also note that because $x_{r}$ lies on a path of maximal length and has distance two from a leaf on that path, every path that connects $x_{r}$ to a leaf and does not contain any other vertex in $P_{d+1}$ must have length at most two. Thus any near leaves on such a path must be directly adjacent to $x_{r}$.

Let $a$ be the number of near leaves adjacent to $x_{r}$ but not on $P_{d+1}$. Then the graph of $I_{r}$ has $p+a$ connected components, and the number of near leaves of $I_{r}$ in the connected component containing $P_{d-2}$ is at least $q-a-1$ when $d \geq 5$, since $y$ is also no longer a near leaf. If $d=4$, the number of near leaves of $I_{r}$ is 0 and $a=q-2$ since every near leaf is adjacent to $x_{r}$, including both that lie on $P_{d+1}$. Hence, for $d=4$, the minor associated to $I_{r}$ consists of $P_{2}$, together with $a$ additional connected components and at least one isolated vertex. Thus

$$
\begin{aligned}
\operatorname{sdepth}\left(S_{r} / I_{r}^{t}\right) & \geq 1+a+p-1+1 \geq q+p-1 \\
& \geq\left\lceil\frac{q+3}{3}\right\rceil+p-1=\left\lceil\frac{d+q-1}{3}\right\rceil+p-1 \geq s
\end{aligned}
$$

for $d=4$ and $q \geq 3$.

Assume that $d=5$. Hence, either the connected component containing $P_{d-2}$ contains two near leaves, and thus has diameter at least $3=d-2$, or $a \geq 1$. In the first case, by induction,

$$
\begin{aligned}
\operatorname{sdepth}\left(S_{r} / I_{r}^{t}\right) & =\operatorname{sdepth}\left(S_{r}^{\prime} / I_{r}^{t}\right)+1 \\
& \geq \max \left\{\left\lceil\frac{d-2-t+q-1}{3}\right\rceil+p-1, p\right\}+1 \geq s .
\end{aligned}
$$

In the second case, $a \geq 1$, and so

$$
\begin{aligned}
\operatorname{sdepth}\left(S_{r} / I_{r}^{t}\right) & =\operatorname{sdepth}\left(S_{r}^{\prime} / I_{r}^{t}\right)+1 \\
& \geq \max \left\{\left\lceil\frac{d-3-t+q-a-1}{3}\right\rceil+p-1, p\right\}+a+1 \geq s .
\end{aligned}
$$


If $d>5$, then $d-2 \geq 4$, and so either $P_{d-2}$ contains two near leaves, one of which was not a near leaf of $I$, or $P_{d-2}$ is not maximal, and so the diameter of $I_{r}$ is at least $d-2$. In the first case, the number of near leaves is $q-a$ and the diameter is at least $d-3$, and so

$$
\begin{aligned}
\operatorname{sdepth}\left(S_{r} / I_{r}^{t}\right) & =\operatorname{sdepth}\left(S_{r}^{\prime} / I_{r}^{t}\right)+1 \\
& \geq \max \left\{\left\lceil\frac{d-3-t+q-a}{3}\right\rceil+p-1, p\right\}+a+1 \geq s
\end{aligned}
$$

In the second case, the number of near leaves is $q-a-1$ and the diameter is at least $d-2$, so again

$$
\begin{aligned}
\operatorname{sdepth}\left(S_{r} / I_{r}^{t}\right) & =\operatorname{sdepth}\left(S_{r}^{\prime} / I_{r}^{t}\right)+1 \\
& \geq \max \left\{\left\lceil\frac{d-2-t+q-a-1}{3}\right\rceil+p-1, p\right\}+a+1 \geq s .
\end{aligned}
$$

Now by reverse induction on $j$, using the proof of Theorem 2.7, we have

$$
\operatorname{sdepth}\left(S_{1} /\left(I_{1}^{t}: y\right)\right)=\operatorname{sdepth}\left(S /\left(\left(I^{t}: y\right), x_{1}\right)\right) \geq s .
$$

As in Theorem 2.7, the result now follows from Lemma 2.4.

\section{ACKNOWLEDGMENT}

The authors would like to thank the referee for a careful reading of the paper and for valuable comments.

\section{REFERENCES}

[1] M. Brodmann, The asymptotic nature of the analytic spread, Math. Proc. Cambridge Philos. Soc. 86 (1979), no. 1, 35-39. MR0530808 (81e:13003)

[2] L. Burch, Codimension and analytic spread, Proc. Cambridge Philos. Soc. 72 (1972), 369373. MR0304377 (46:3512)

[3] M. Cimpoeas, Some remarks on the Stanley depth for multigraded modules, Matematiche (Catania) 63 (2008), no. 2, 165-171. MR2531658(2010d:13032)

[4] S. Morey, Depths of powers of the edge ideal of a tree, Comm. Algebra 38 (2010), no. 11, 4042-4055. MR2764849 (2011m:13039)

[5] M. R. Pournaki, S. A. Seyed Fakhari, M. Tousi, S. Yassemi, What is .. Stanley depth? Notices Amer. Math. Soc. 56 (2009), no. 9, 1106-1108. MR2568497 (2010k:05346)

[6] A. Rauf, Stanley decompositions, pretty clean filtrations and reductions modulo regular elements, Bull. Math. Soc. Sci. Math. Roumanie (N.S.) 50(98) (2007), no. 4, 347-354. MR2370321 (2008i:13035)

[7] A. Rauf, Depth and Stanley depth of multigraded modules, Comm. Algebra 38 (2010), no. 2, 773-784. MR2598911 (2011g:13029)

[8] A. Simis, W. V. Vasconcelos, R. H. Villarreal, On the ideal theory of graphs, J. Algebra $\mathbf{1 6 7}$ (1994), no. 2, 389-416. MR.1283294 (95e:13002)

[9] R. P. Stanley, Linear Diophantine equations and local cohomology, Invent. Math. 68 (1982), no. 2, 175-193. MR0666158(83m:10017)

[10] W. Vasconcelos, Integral Closure, Rees Algebras, Multiplicities, Algorithms, Springer Monographs in Mathematics. Springer-Verlag, Berlin, 2005. MR2153889 (2006m:13007) 
Department of Mathematical Sciences, Sharif University of Technology, P.O. Box 11155-9415, Tehran, Iran - And - School of Mathematics, Institute for Research iN Fundamental Sciences (IPM), P.O. Box 19395-5746, Tehran, Iran

E-mail address: pournaki@ipm.ir

$U R L:$ http://math.ipm.ac.ir/pournaki/

Department of Mathematical Sciences, Sharif University of Technology, P.O. Box 11155-9415, TEHRAN, IRAN

E-mail address: fakhari@ipm.ir

$U R L:$ http://math.ipm.ac.ir/fakhari/

School of Mathematics, Statistics and Computer Science, College of Science, University of Tehran, Tehran, Iran - and - School of Mathematics, Institute for Research in Fundamental Sciences (IPM), P.O. Box 19395-5746, Tehran, Iran

E-mail address: yassemi@ipm.ir

URL: http://math.ipm.ac.ir/yassemi/ 